Supporting Information for

\title{
Resistive Switching Memory Performance of Two- \\ Dimensional Polyimide Covalent Organic Framework Films
}

\author{
Bing Sun, ${ }^{\dagger,}, *$ Xinle Li, ${ }^{\ddagger}$ Tiantian Feng, ${ }^{\dagger, \S}$ Songliang Cai,,$"$, Teresa Chen,${ }^{\ddagger}$ Chenhui \\ Zhu, ${ }^{\perp}$ Jian Zhang, ${ }^{\ddagger}$ Dong Wang,,${ }^{*}$ Yi Liu, \\ $†$ School of Science, China University of Geosciences (Beijing), Beijing 100083, P.R. China \\ † The Molecular Foundry, Lawrence Berkeley National Laboratory, Berkeley, CA 94720, USA \\ $\S$ Key Laboratory of Molecular Nanostructure and Nanotechnology, Institute of Chemistry, Chinese \\ Academy of Sciences, Beijing 100190, P.R. China \\ School of Chemistry, South China Normal University, Guangzhou 510006, P.R. China \\ $\perp$ Advanced Light Source, Lawrence Berkeley National Laboratory, Berkeley, CA 94720, USA
}

*Corresponding authors:

E-mails: yliu@lbl.gov (Y.L.); sunbing@cugb.edu.cn (B.S.); wangd@iccas.ac.cn (D.W.)

ORCID:

Bing Sun: 0000-0001-5917-3094

Songliang Cai: 0000-0002-5399-9036

Dong Wang: 0000-0002-1649-942X

Yi Liu: 0000-0002-3954-6102 


\section{S1. Synthesis and characterization of PI-NT COF powder}

The PI-NT-COF powder was synthesized via a solvothermal method. Typically, TAPA (58 mg, $0.2 \mathrm{mmol}$ ), NTCDA (80.4 mg, $0.3 \mathrm{mmol})$, mesitylene (1.5 mL), NMP $(1.5 \mathrm{~mL})$ and isoquinoline $(0.2 \mathrm{~mL})$ were charged in a PTFE autoclave chamber $(25 \mathrm{~mL}$ in total capacity), and ultrasonically treated for $15 \mathrm{~min}$ to obtain a well-dispersed suspension and deaerated with inert nitrogen gas for $15 \mathrm{~min}$. After being sealed in the corresponding stainless-steel container, the reactor was heated at $200{ }^{\circ} \mathrm{C}$ for 3 days. The precipitated brown powder was collected via centrifugation after the reaction was cooled down to room temperature, and then was washed by THF and dried under vacuum.

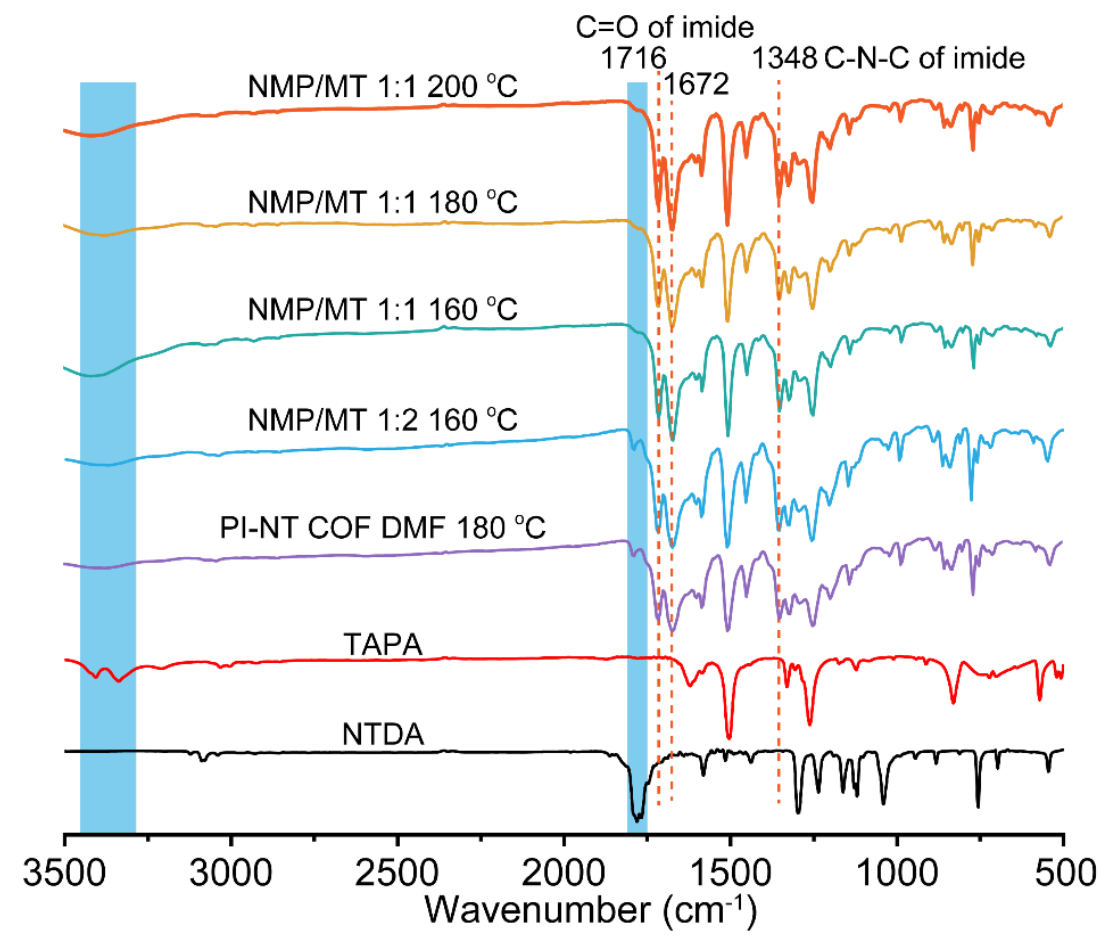

Figure S1. FTIR spectra of PI-NT COF powder synthesized under various conditions. (MT=mesitylene)

In the FTIR spectrum of PI-NT COF powder, the stretching peaks of amino groups in TAPA in the range from 3300 to $3450 \mathrm{~cm}^{-1}$ and $\mathrm{C}=\mathrm{O}$ groups in NTCDA around 1775 $\mathrm{cm}^{-1}$ disappeared, indicating the full consumption of monomers in the polycondensation reaction. In contrast, peaks corresponding to residual anhydride $\mathrm{C}=\mathrm{O}$ groups were observed in the products obtained from the reported synthetic conditions using DMF 
and NMP/mesitylene $(1: 2 \mathrm{v} / \mathrm{v})$ as the solvent. The emerging characteristic peaks at 1716 and $1672 \mathrm{~cm}^{-1}$ were ascribed to the asymmetric stretching of imide $\mathrm{C}=\mathrm{O}$ and the peak at $1348 \mathrm{~cm}^{-1}$ was attributed to the stretching mode of imide $\mathrm{C}-\mathrm{N}-\mathrm{C}$, supporting the formation of imide groups in PI-NT COF powder.

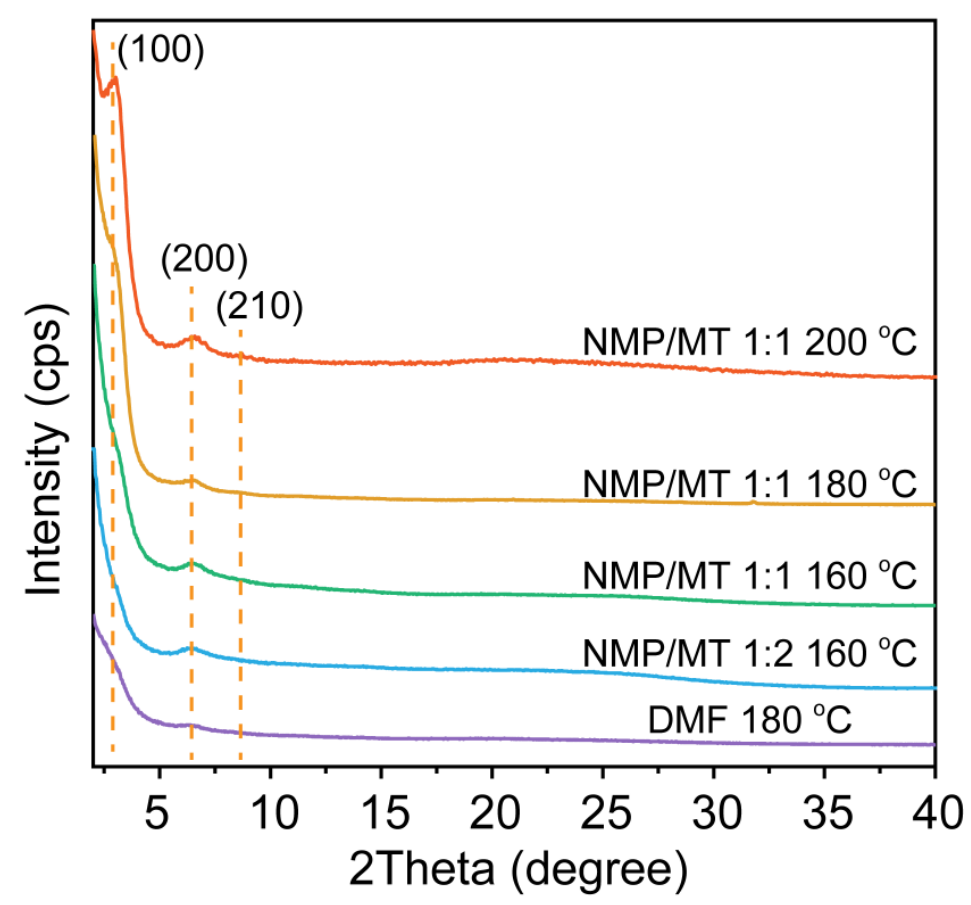

Figure S2. PXRD patterns of PI-NT COF powders synthesized under various conditions.

Powder X-ray diffraction (PXRD) was used to optimize the synthetic conditions and confirm the structural regularity. The PXRD patterns (Figure S3) showed increasing number of diffraction peaks as the solvents switched from NMP/mesitylene (1:2) and DMF to NMP/mesitylene (1:1), and the intensity of these main peaks further increased when raising the reaction temperature to $200{ }^{\circ} \mathrm{C}$. Therefore, NMP/mesitylene (1:1) with isoquinoline as the catalyst and $200{ }^{\circ} \mathrm{C}$ are utilized for the synthesis of PINT COF powder. 


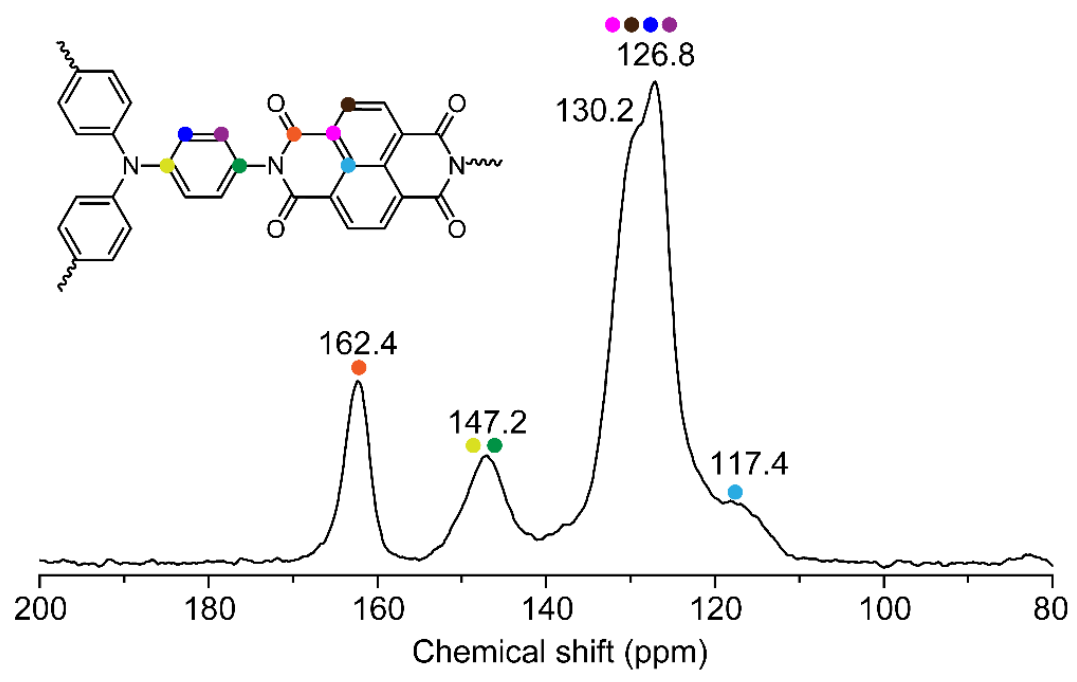

Figure S3. Solid-state NMR spectrum of PI-NT COF powder constructed from TAPA and NTCDA.

The chemical identity of PI-NT COF powder was further confirmed by solid-state ${ }^{13} \mathrm{C}$ NMR spectroscopy. The signal around $162.4 \mathrm{ppm}$ in the ${ }^{13} \mathrm{C}$ NMR spectrum (Figure S2) could be assigned to the imide carbon in the PI-NT COF powder, and the other peaks with the chemical shifts of 117.4, 126.8, 130.2 and 147.2 ppm were assigned to the aromatic carbons. 


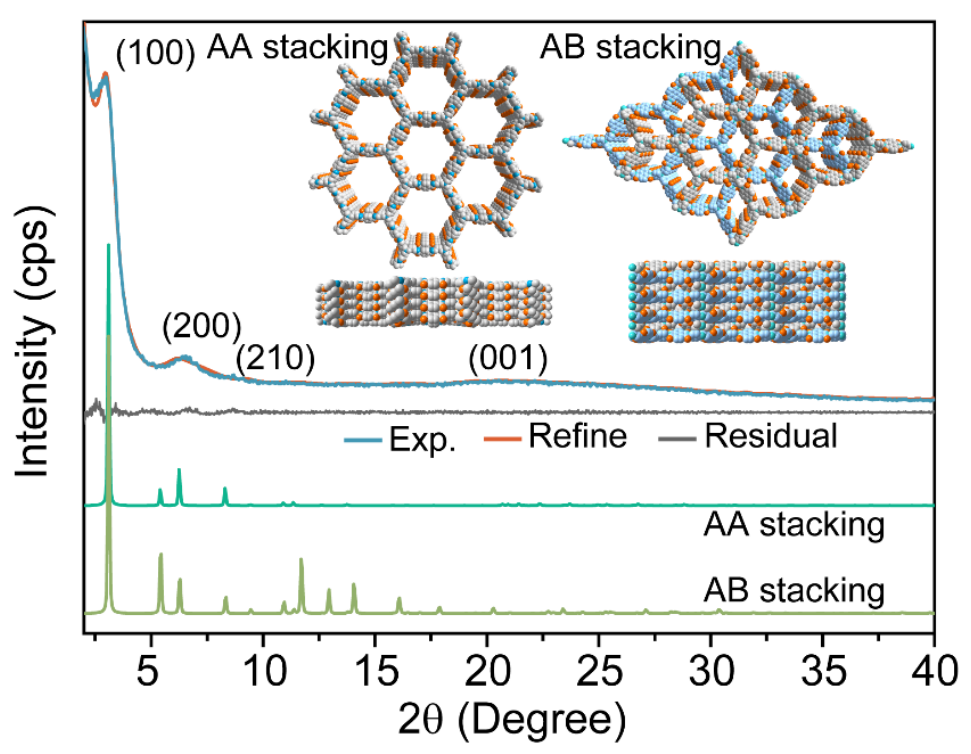

Figure S4. Experimental, simulated AA stacking, simulated AB stacking, Pawley refined PXRD patterns of PI-NT COF powder and the difference (residual) between experimental and refined patterns. Inset: top-view and side-view illustrations of simulated AA and AB stacking models for PI-NT COF.

Based on the monomer structures and imide bonding motifs between TAPA and NTCDA building blocks, a two-dimensional layered bnn topology with a hexagonal aperture of $2.6 \mathrm{~nm}$ was proposed for the simulated pattern. The eclipsed (AA, space group of $P-31 \mathrm{~m})$ and staggered (AB, space group of $P-31 \mathrm{c}$ ) stacking models (Figure $\mathrm{S} 4$, inset) were optimized using the universal force field. The Pawley refinement demonstrated that the AA stacking model gave the best fit, and the calculated unit cell parameters were obtained with good residual factors: $a=b=32.2430 \AA, c=4.2758 \AA$, $\alpha=\beta=90^{\circ}, \gamma=120^{\circ}, R_{\mathrm{wp}}=4.73 \%$ and $R_{\mathrm{p}}=3.47 \%$. 


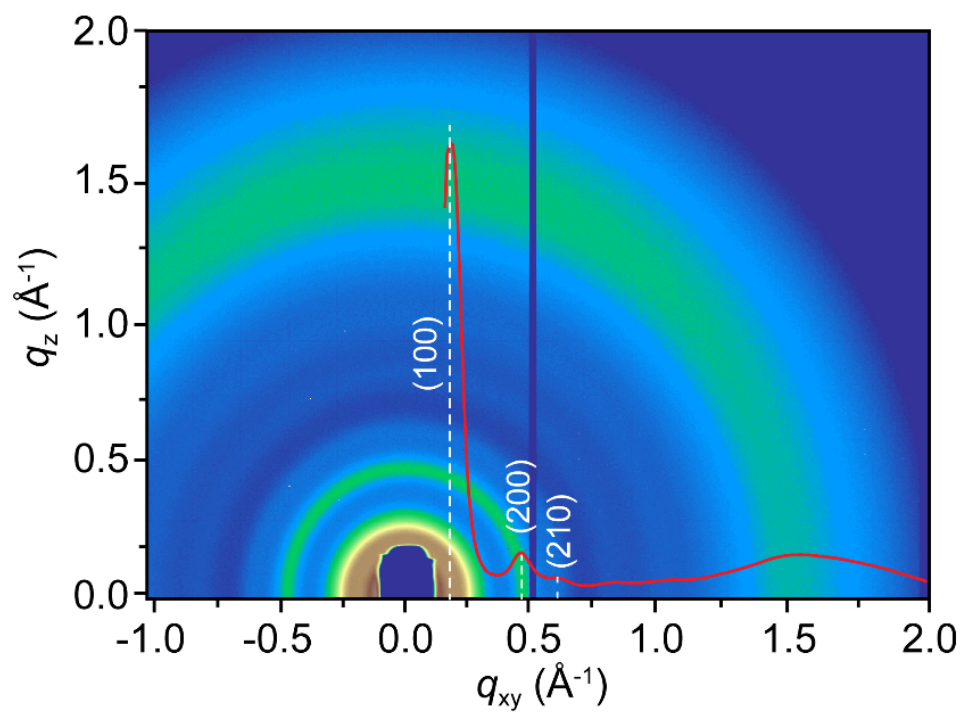

Figure S5. Synchrotron wide-angle diffraction pattern of PI-NT COF powder overlaid with the line profile plot (red curve). 
S2. Synthesis and characterization of PI-NT COF film on ITO-coated glass substrate
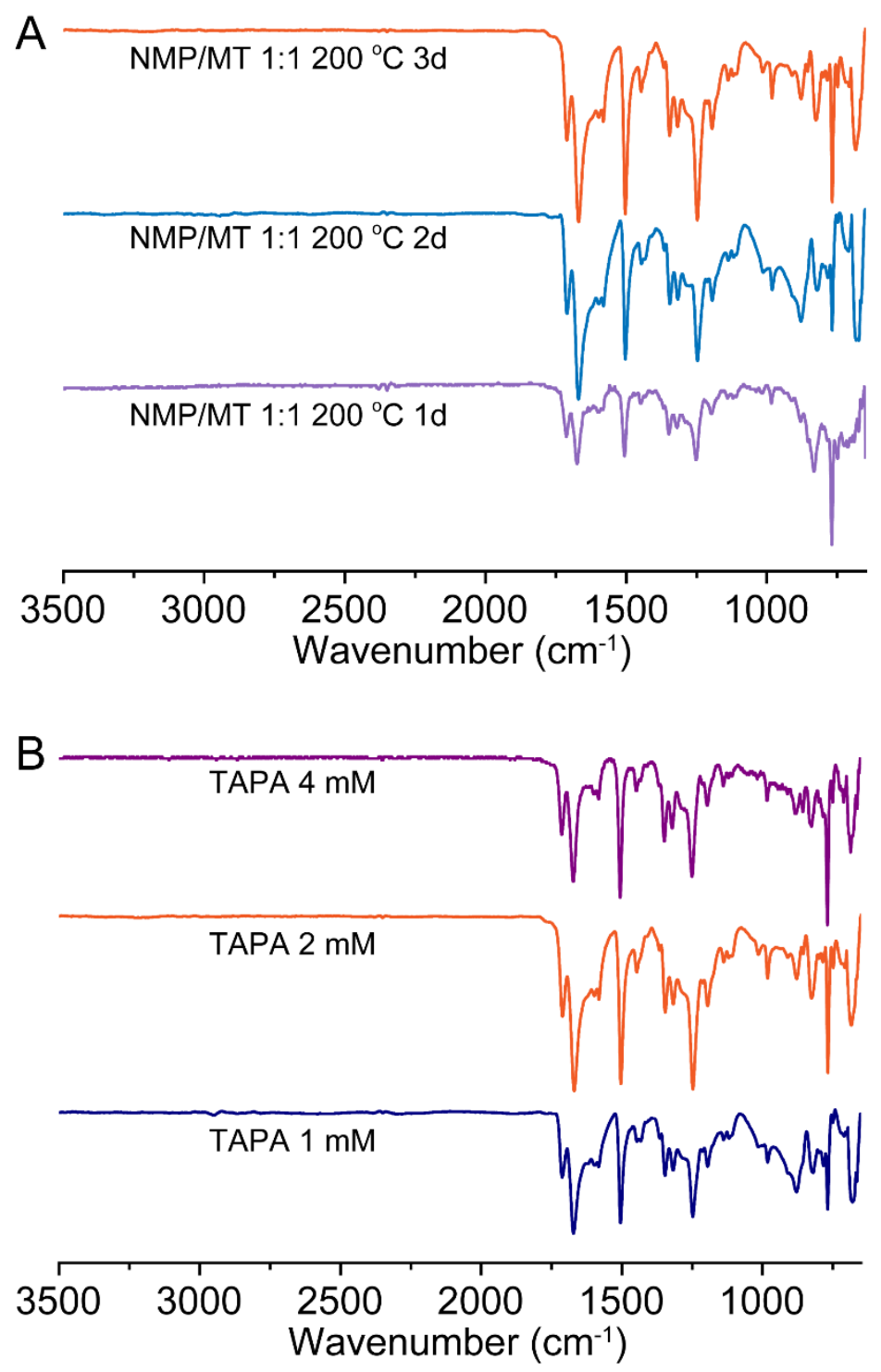

Figure S6. (A) Time-dependent ATR-FTIR spectra of PI-NT COF films made from 2 mM TAPA and $3 \mathrm{mM}$ NTCDA. (B) ATR-FTIR spectra of PI-NT COF films with different monomer concentrations (based on that of TAPA) synthesized at $200{ }^{\circ} \mathrm{C}$ for $3 \mathrm{~d}$ in the solvent of NMP/mesitylene $(1: 1, \mathrm{v} / \mathrm{v})$. 

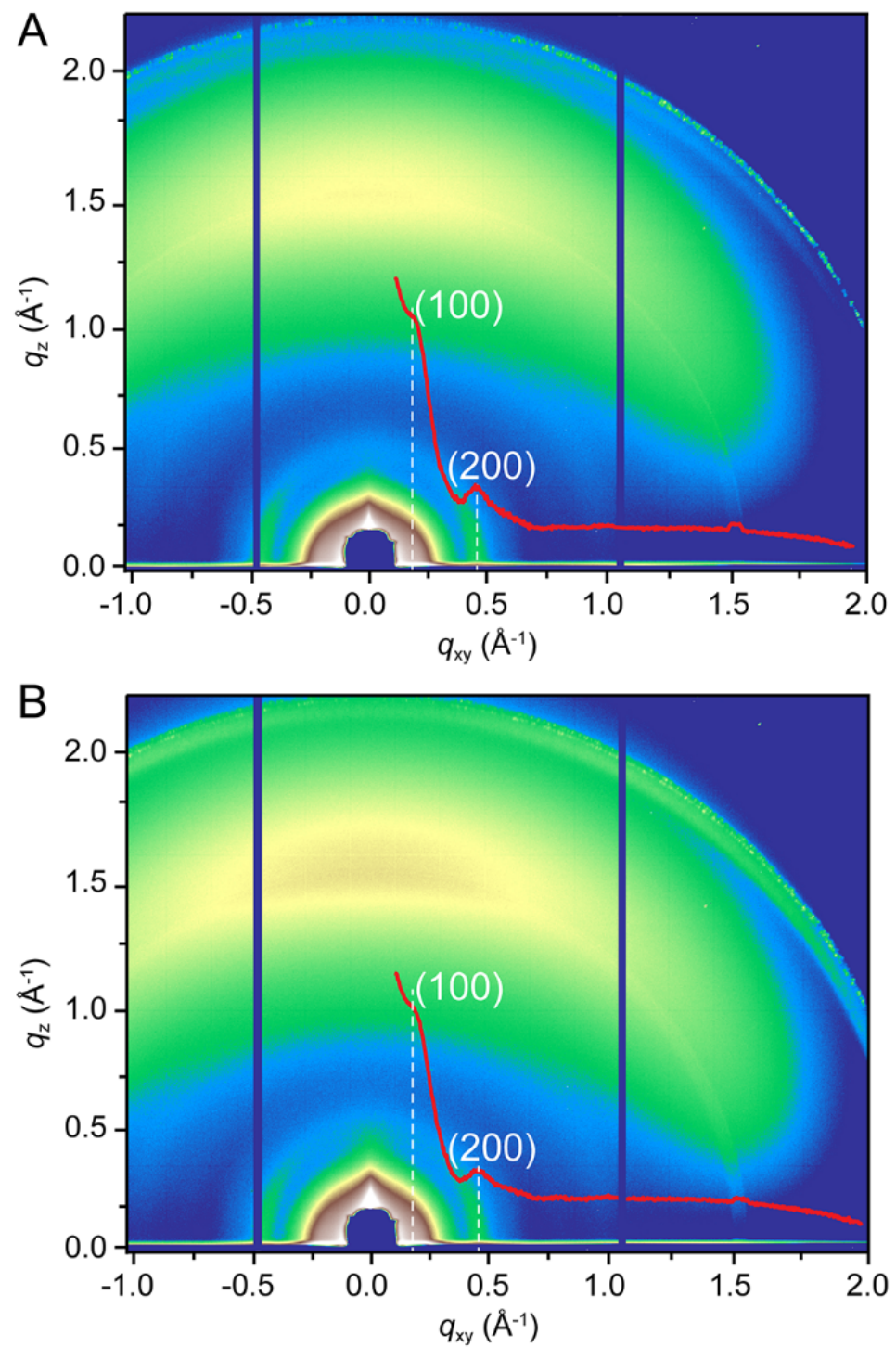

Figure S7. GIWAXS patterns and in-plane line profiles of PI-NT COF films synthesized from the solvent of $\mathrm{NMP} /$ mesitylene $(1: 1, \mathrm{v} / \mathrm{v})$ at $200{ }^{\circ} \mathrm{C}$ for (A) $1 \mathrm{~d}$ and (B) $2 \mathrm{~d}$. 

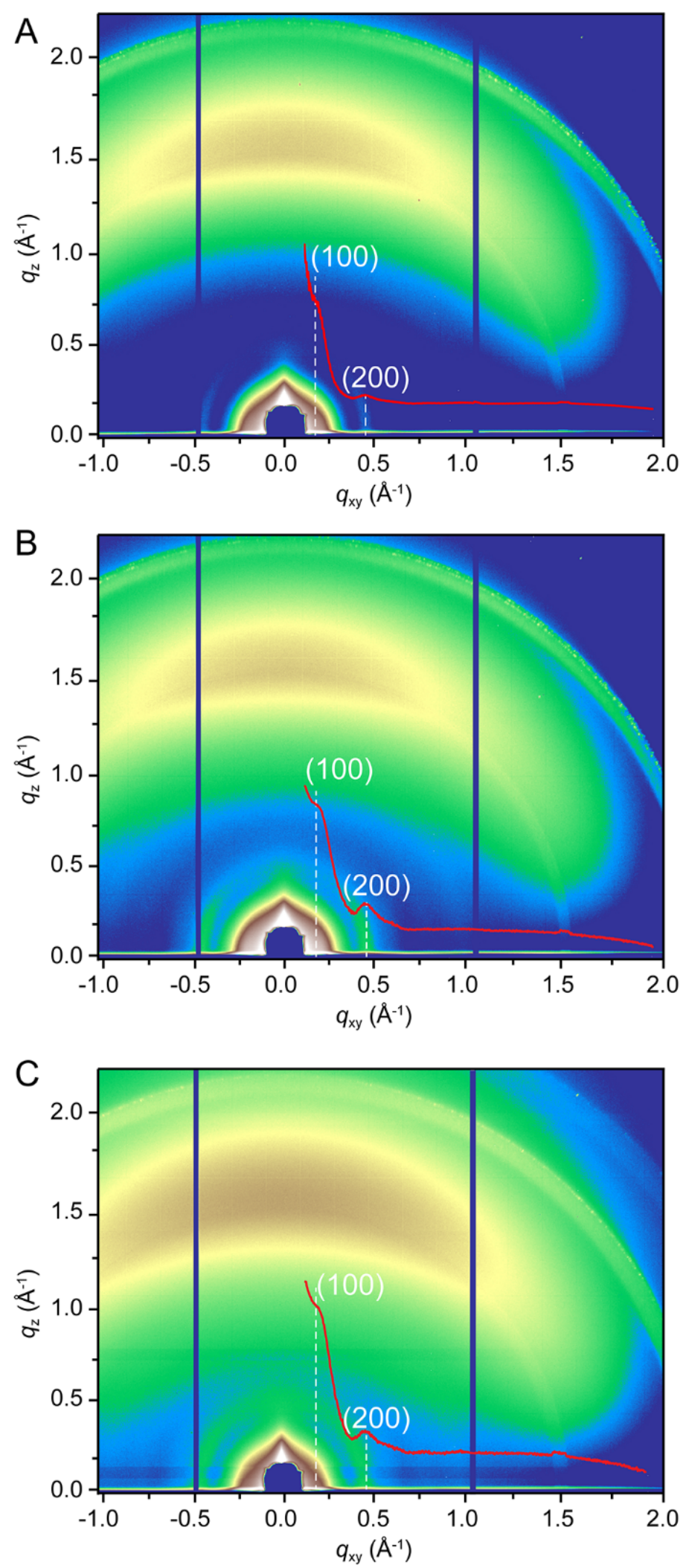

Figure S8. GIWAXS patterns and in-plane line profiles of PI-NT COF films at the monomer concentrations of (A) $1 \mathrm{mM}$ TAPA and $1.5 \mathrm{mM}$ NTCDA, (B) $2 \mathrm{mM}$ TAPA and $3 \mathrm{mM}$ NTCDA, and (C) $4 \mathrm{mM}$ TAPA and $6 \mathrm{mM}$ NTCDA from the solvent of $\mathrm{NMP} /$ mesitylene $(1: 1, \mathrm{v} / \mathrm{v})$ at $200^{\circ} \mathrm{C}$ for $3 \mathrm{~d}$. 

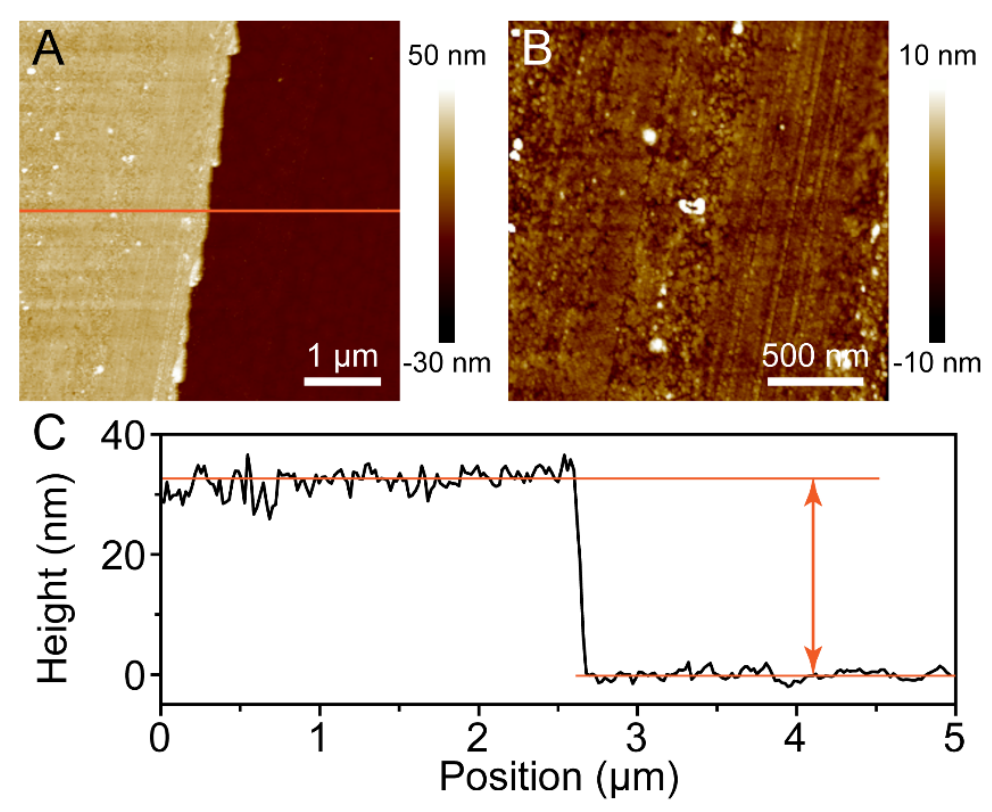

Figure S9. Topographic images of PI-NT COF films (A) at edge and (B) on surface with the monomer concentration of $1 \mathrm{mM}$ TAPA and $1.5 \mathrm{mM}$ NTCDA from the solvent of NMP/mesitylene $(1: 1, \mathrm{v} / \mathrm{v})$ at $200{ }^{\circ} \mathrm{C}$ for $3 \mathrm{~d}$. (C) The cross-section height profile.

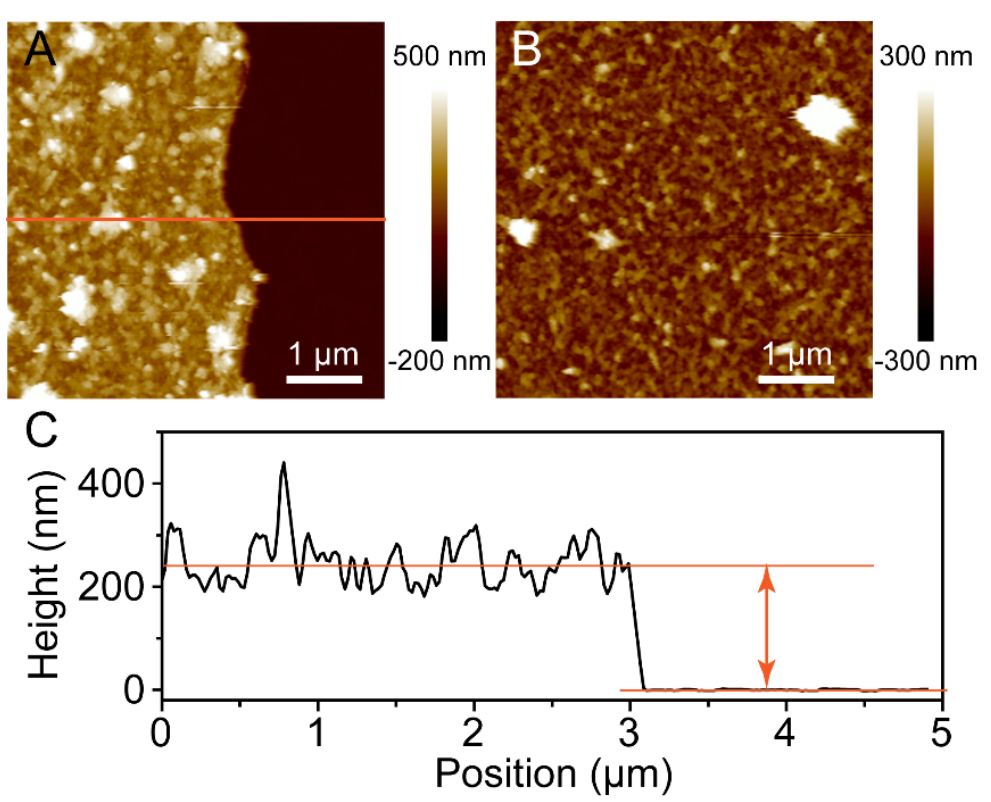

Figure S10. Topographic images of PI-NT COF films (A) at edge and (B) on surface with the monomer concentration of $4 \mathrm{mM}$ TAPA and $6 \mathrm{mM}$ NTCDA in the solvent of $\mathrm{NMP} / \mathrm{mesitylene}(1: 1, \mathrm{v} / \mathrm{v})$ at $200^{\circ} \mathrm{C}$ for $3 \mathrm{~d}$. (C) The cross-section height profile. 
Table S1. Concentration-dependent thickness and surface roughness of PI-NT COF films on ITO.

\begin{tabular}{cccc}
\hline Entry & $\begin{array}{c}\text { Monomer concentration } \\
\text { (based on TAPA) }\end{array}$ & $\begin{array}{c}\text { Thickness of PI- } \\
\text { COF films }\end{array}$ & $\begin{array}{c}\text { Surface } \\
\text { roughness (Ra) }\end{array}$ \\
\hline $\mathbf{1}$ & $1 \mathrm{mM}$ & $38 \pm 7 \mathrm{~nm}$ & $1.63 \mathrm{~nm}$ \\
$\mathbf{2}$ & $2 \mathrm{mM}$ & $102 \pm 12 \mathrm{~nm}$ & $2.26 \mathrm{~nm}$ \\
$\mathbf{3}$ & $4 \mathrm{mM}$ & $274 \pm 71 \mathrm{~nm}$ & $54.6 \mathrm{~nm}$ \\
\hline
\end{tabular}




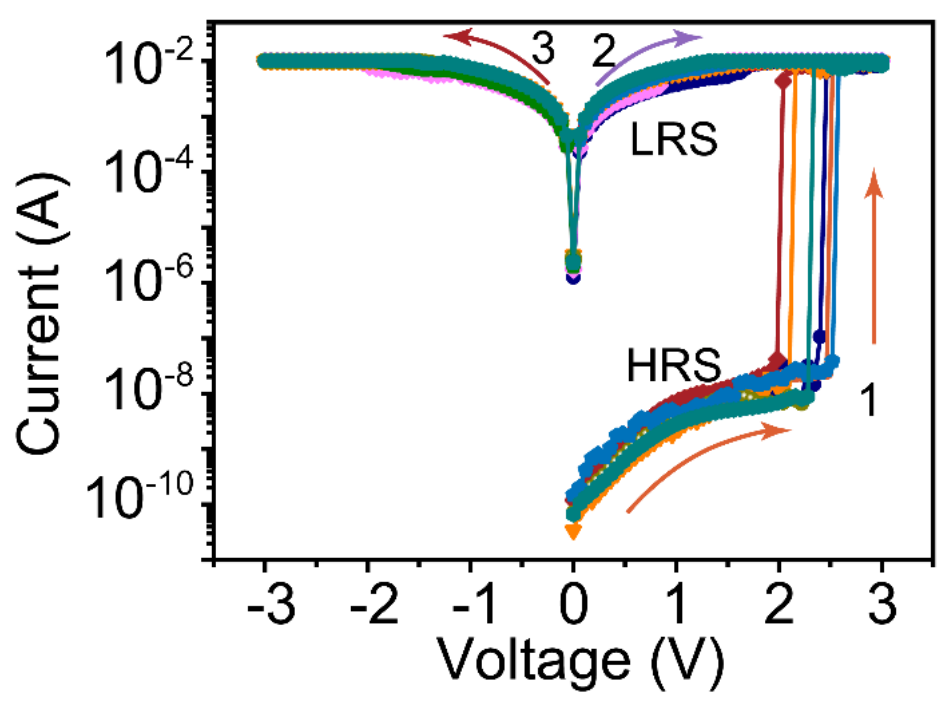

Figure S11. An overlay of several $I-V$ characteristics from the positive scans for different $\mathrm{Al} / \mathrm{LiF} / \mathrm{PI}-\mathrm{NT}$ COF film/ITO devices. The number and arrows represented the voltage sweeping order and directions.

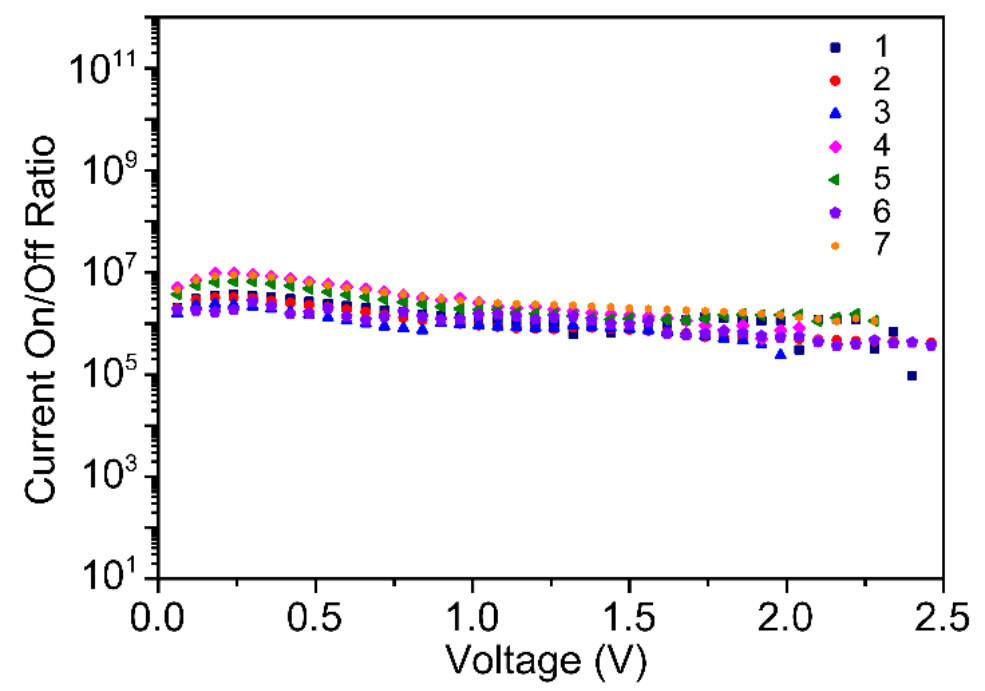

Figure S12. Current on/off ratio of seven memory devices. 


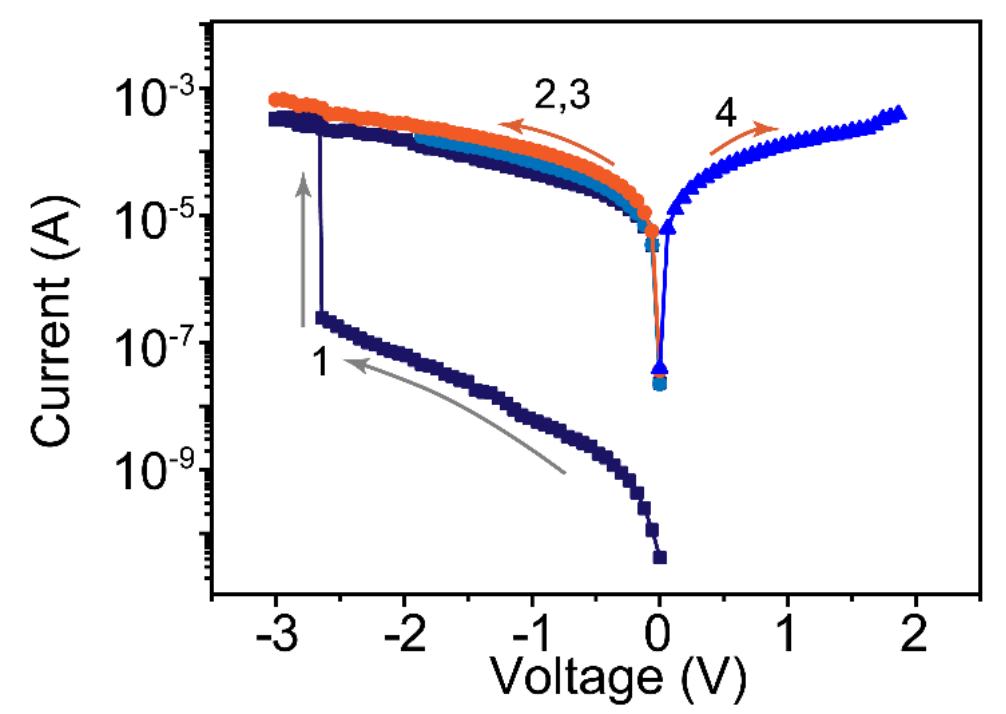

Figure S13. $I-V$ characteristics on the negative scans for the $\mathrm{Al} / \mathrm{LiF} / \mathrm{PI}-\mathrm{NT}$ COF film/ITO devices. The number and arrows represented the voltage sweeping order and directions. 
Table S2. Comparison of performances of resistive memory devices fabricated based on diimide-type D-A small molecules and polymers.

\begin{tabular}{|c|c|c|c|c|c|c|}
\hline $\begin{array}{l}\text { Active layer materials } \\
\text { [Classification] }\end{array}$ & Memory devices & Memory effect & Switching mechanism & Switching voltage & $\begin{array}{c}\text { ON/OFF } \\
\text { current ratio }\end{array}$ & Ref \\
\hline $\begin{array}{c}\text { NONIBTDT } \\
{[\text { D-A small molecule] }}\end{array}$ & ITO/NONIBTDT/Al & $\begin{array}{l}\text { quaternary } \\
\text { WORM }\end{array}$ & $\begin{array}{l}\text { Electric-field-induced } \\
\text { charge transfer }\end{array}$ & $\begin{array}{l}-2.04 \mathrm{~V} \\
-2.73 \mathrm{~V} \\
-3.96 \mathrm{~V}\end{array}$ & $\begin{array}{l}10^{1.78} \\
10^{3.47} \\
10^{5.36}\end{array}$ & S1 \\
\hline $\begin{array}{l}\text { AsPDIs (PDI-ATPA and PDI- } \\
\text { TATPA) } \\
\text { [D-A small molecules] }\end{array}$ & ITO/PDI-ATPA/Al & $\begin{array}{l}\text { WORM } \\
\text { SRAM }\end{array}$ & $\begin{array}{l}\text { Electric-field-induced } \\
\text { charge transfer }\end{array}$ & $\begin{array}{l}1.4 \mathrm{~V}(2.7 \mathrm{~V}) \\
2.7 \mathrm{~V} / 3.2 \mathrm{~V}\end{array}$ & $\begin{array}{c}10^{6} \\
10^{3} \sim 10^{4}\end{array}$ & S2 \\
\hline $\begin{array}{c}\text { TPA-3T-NI } \\
\text { [D-A small molecule] }\end{array}$ & Al/TPA-3T-NI/Al & WORM & $\begin{array}{l}\text { Electric-field-induced } \\
\text { charge transfer }\end{array}$ & $-1.4 \mathrm{~V}$ & $\sim 10^{4}$ & S3 \\
\hline $\begin{array}{c}\text { DBIP (TPA-imidazole-[4,5- } \\
\text { b]phenazine) } \\
\text { [D-A small molecule] }\end{array}$ & ITO/DBIP/Pt & rewritable & $\begin{array}{l}\text { Electric-field-induced } \\
\text { charge transfer }\end{array}$ & $2.25 \mathrm{~V}$ & $10^{3}$ & S4 \\
\hline $\begin{array}{c}\text { TPA-2BIPs, TPA-3BIPs) } \\
\text { [D-A small molecules] }\end{array}$ & $\begin{array}{c}\text { ITO/TPA-2BIPs (or TPA- } \\
\text { 3BIPs) } / \mathrm{Au}\end{array}$ & rewritable & $\begin{array}{l}\text { Electric-field-induced } \\
\text { charge transfer }\end{array}$ & $\begin{array}{l}0.83 \mathrm{~V} \\
0.84 \mathrm{~V}\end{array}$ & $\begin{array}{l}10^{3} \\
10^{2}\end{array}$ & S5 \\
\hline $\begin{array}{c}\text { Polyazothines } \\
{[\text { D-A poly(Schiff base)] }}\end{array}$ & $\begin{array}{l}\mathrm{Pt} / \mathrm{PA}-1 / \mathrm{Pt} \\
\mathrm{Pt} / \mathrm{PA}-1 / \mathrm{Al} \\
\mathrm{Pt} / \mathrm{PA}-2 / \mathrm{Pt} \\
\mathrm{Pt} / \mathrm{PA}-2 / \mathrm{Al}\end{array}$ & $\begin{array}{c}\text { rewritable } \\
\text { rewritable } \\
\text { WORM } \\
\text { rewritable }\end{array}$ & $\begin{array}{l}\text { Electric-field-induced } \\
\text { charge transfer }\end{array}$ & $\begin{array}{l}-3.3 \mathrm{~V} \\
-2.2 \mathrm{~V} \\
-4.6 \mathrm{~V} \\
-2.5 \mathrm{~V}\end{array}$ & $\begin{array}{c}>10^{5} \\
\sim 10^{5} \\
\text { Up to } 10^{7} \\
3 \times 10^{4}\end{array}$ & S6 \\
\hline $\begin{array}{c}\text { PFcFE1 } \\
\text { [TPA-based polymer] }\end{array}$ & ITO/PFcFE1/Al & rewritable & Redox & $-1.0 \mathrm{~V}$ & $10^{3}$ & S7 \\
\hline
\end{tabular}




\begin{tabular}{|c|c|c|c|c|c|c|}
\hline $\begin{array}{l}\text { Active layer materials } \\
\text { [Classification] }\end{array}$ & Memory devices & Memory effect & Switching mechanism & Switching voltage & $\begin{array}{l}\text { ON/OFF } \\
\text { current ratio }\end{array}$ & Ref \\
\hline $\begin{array}{l}\text { Poly[N,N-bis(4- } \\
\text { aminophenyl)aminopyrene- } \\
\text { hexafluoroisopropylidene- } \\
\text { diphthalimide] PI(APAP) } \\
\text { [D-A linear polymer] }\end{array}$ & Al/PI(APAP)/Al & WORM & $\begin{array}{l}\text { Electric-field-induced } \\
\text { charge transfer }\end{array}$ & $-3.0 \mathrm{~V}$ & $>10^{4}$ & S8 \\
\hline $\begin{array}{c}\mathrm{PTPA}_{2} \mathrm{OXD}_{8} \\
{[\mathrm{D}-\mathrm{A} \text { co-polymer }]}\end{array}$ & $\mathrm{ITO} / \mathrm{PTPA}_{2} \mathrm{OXD}_{8} / \mathrm{Al}$ & WORM & $\begin{array}{l}\text { Electric-field-induced } \\
\text { charge transfer }\end{array}$ & $2.4 \mathrm{~V}$ & $1.7 \times 10^{3}$ & S9 \\
\hline $\begin{array}{l}\text { PI(AMTPA):coronene and } \\
\text { PI(AMTPA):PDI-DO blend } \\
\text { thin film } \\
\text { [D-A polymer blend] }\end{array}$ & $\begin{array}{l}\text { Al/PI(AMTPA): } 10 \% \\
\text { coronene/Al } \\
\text { Al/PI(AMTPA): } \\
10 \% \text { PDI-DO/Al }\end{array}$ & $\begin{array}{l}\text { WORM } \\
\text { WORM }\end{array}$ & $\begin{array}{l}\text { Electric-field-induced } \\
\text { charge transfer }\end{array}$ & $\begin{array}{l}-2.4 \mathrm{~V} \\
\sim-2.4 \mathrm{~V}\end{array}$ & $\begin{array}{l}>10^{4} \\
\sim 10^{3}\end{array}$ & $\mathrm{~S} 10$ \\
\hline $\begin{array}{l}\text { PI-6FDA and CoPI-6FDA } \\
\text { [D-A imide polymer] }\end{array}$ & $\begin{array}{c}\text { ITO/PI-6FDA/Al } \\
\text { ITO/CoPI-6FDA/Al }\end{array}$ & WORM & $\begin{array}{l}\text { Electric-field-induced } \\
\text { charge transfer }\end{array}$ & $\begin{array}{l}-3.5 \mathrm{~V} \\
-3.1 \mathrm{~V}\end{array}$ & $\begin{array}{l}10^{4} \\
10^{5}\end{array}$ & S11 \\
\hline $\begin{array}{l}\text { Poly(siloxane-imide)s } \\
\text { [D-A imide polymer] }\end{array}$ & $\begin{array}{c}\text { PDMS/CNTs/ODPA- } \\
\text { A12/Al }\end{array}$ & WORM & $\begin{array}{l}\text { Electric-field-induced } \\
\text { charge transfer }\end{array}$ & $\begin{array}{l}\sim 3.2 \mathrm{~V} \\
\sim 2.5 \mathrm{~V}\end{array}$ & $\begin{array}{l}10^{4} \sim 10^{5} \\
>10^{6}\end{array}$ & S12 \\
\hline
\end{tabular}




\begin{tabular}{|c|c|c|c|c|c|c|}
\hline $\begin{array}{c}\text { Active layer materials } \\
\text { [Classification] }\end{array}$ & Memory devices & Memory effect & Switching mechanism & Switching voltage & $\begin{array}{l}\text { ON/OFF } \\
\text { current ratio }\end{array}$ & Ref \\
\hline $\begin{array}{l}\text { Aromatic polyimide } \\
\quad \text { (BTDBPI) } \\
\text { [D-A imide polymer] }\end{array}$ & ITO/BTDBPI $(60 \mathrm{~nm}) / \mathrm{Au}$ & WORM & $\begin{array}{l}\text { Electric-field-induced } \\
\text { charge transfer }\end{array}$ & $2.3 \mathrm{~V}$ & $10^{4}$ & S13 \\
\hline $\begin{array}{c}\text { porphyrin-containing PI, } \\
\text { ZnPor-t-DSDA } \\
\text { [D-A imide polymer] }\end{array}$ & $\begin{array}{c}\text { ITO/ZnPor-t-DSDA/Au or } \\
\mathrm{Al}\end{array}$ & DRAM & $\begin{array}{l}\text { Electric-field-induced } \\
\text { charge transfer }\end{array}$ & $\begin{array}{l}3.9 \mathrm{~V} \text { for positive } \\
\text { scan } \\
-3.9 \mathrm{~V} \text { for negative } \\
\text { scan }\end{array}$ & $\sim 10^{6}$ & S14 \\
\hline $\begin{array}{l}\text { P(INDISi-alt-CzSi) } \\
\text { [D-A polymer] }\end{array}$ & $\begin{array}{c}\mathrm{PET} / \mathrm{ITO} / \mathrm{P}(\mathrm{INDISi} \text {-alt- } \\
\mathrm{CzSi}) / \mathrm{Al}\end{array}$ & $\begin{array}{l}\text { Ternary } \\
\text { WORM }\end{array}$ & $\begin{array}{l}\text { Electric-field-induced } \\
\text { charge transfer }\end{array}$ & $\begin{array}{l}\text { ON1 } 0.8 \mathrm{~V} \\
\text { ON2 } 2.5 \mathrm{~V}\end{array}$ & $\sim 10^{5}$ & S15 \\
\hline $\begin{array}{l}\text { 2D imine polymer (2DP) film } \\
\text { [2D COF-like polymer] }\end{array}$ & $\mathrm{Ag} / 2 \mathrm{DP} / \mathrm{ITO}$ & rewritable & Filament & $\sim 0.90 \mathrm{~V}$ & $>10^{5}$ & S16 \\
\hline PI-NT COF film & $\begin{array}{l}\text { ITO/PI-NT COF } \\
\text { film/LiF/Al }\end{array}$ & WORM & $\begin{array}{l}\text { Electric-field-induced } \\
\text { charge transfer }\end{array}$ & $\begin{array}{l}2.4 \mathrm{~V} \text { for positive } \\
\text { scan } \\
-2.64 \mathrm{~V} \text { for negative } \\
\text { scan }\end{array}$ & $\begin{array}{c}>10^{6} \\
10^{4} \sim 10^{6}\end{array}$ & $\begin{array}{l}\text { This } \\
\text { work }\end{array}$ \\
\hline
\end{tabular}

Notes: WORM: write-once-read-many times memory (non-volatile); SARM: static random access memory (volatile); DRAM: dynamic random access memory (volatile). 

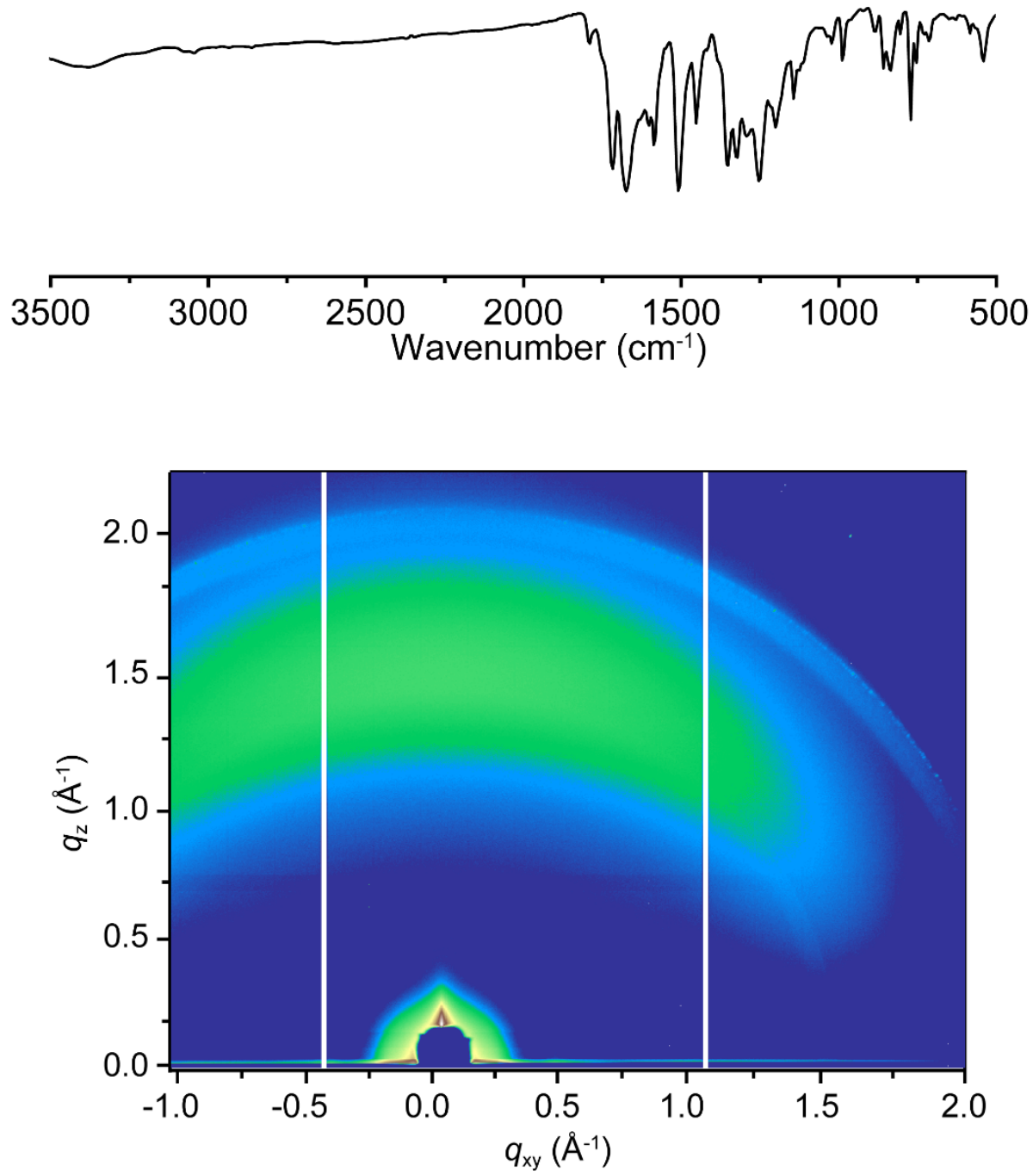

Figure S14. Characterization of PI-NT amorphous films on ITO substrates. 

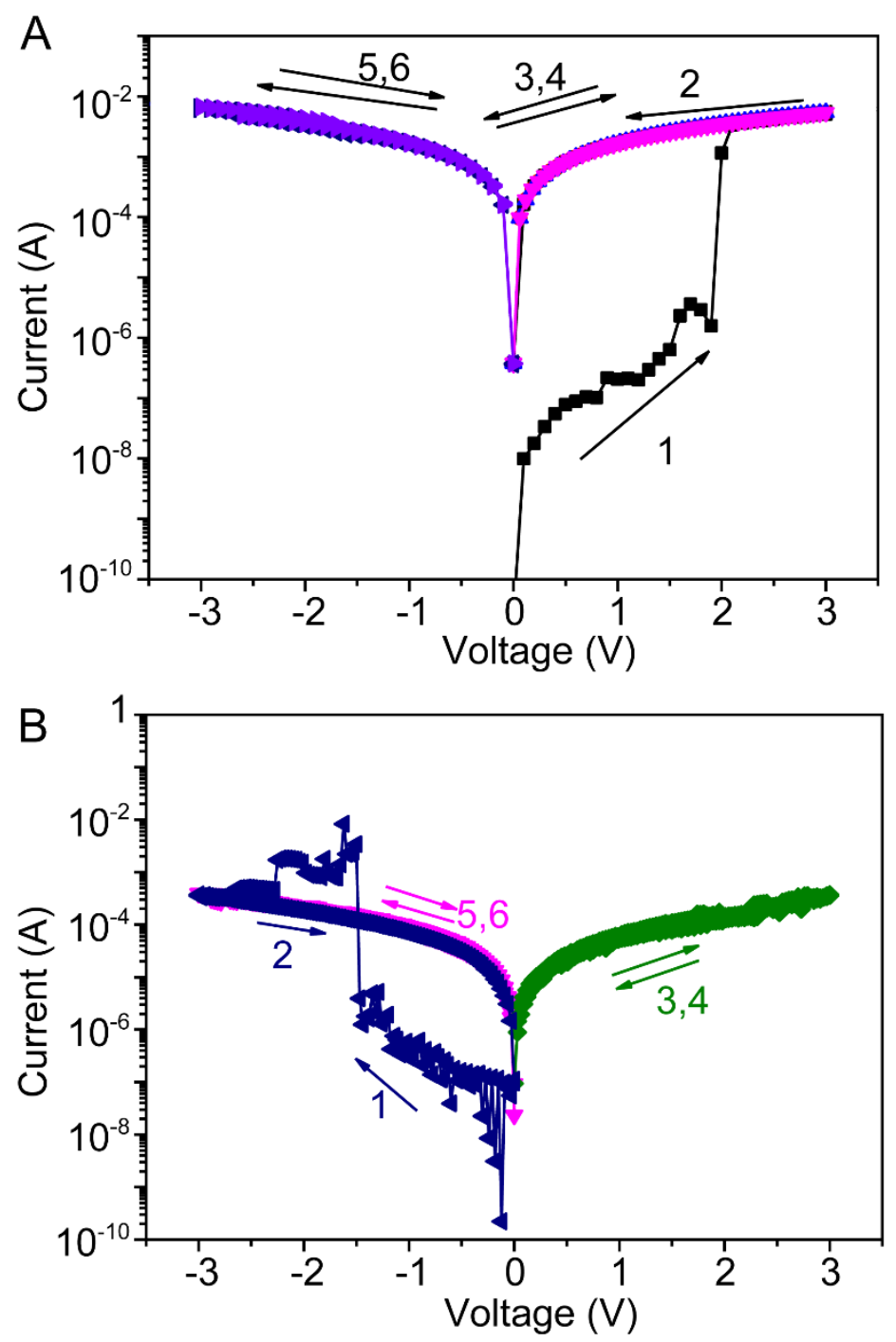

Figure S15. (A) $I-V$ characteristics of the ITO/PI-NT amorphous film $/ \mathrm{LiF} / \mathrm{Al}$ on the positive scan. Sweep 1: from $0 \mathrm{~V}$ to $+3 \mathrm{~V}$; sweeps 2: from $+3 \mathrm{~V}$ to $0 \mathrm{~V}$; sweeps 3 and 4: reversible sweep between $0 \mathrm{~V}$ to $+3 \mathrm{~V}$; sweeps 5 and 6: reversible sweep between $0 \mathrm{~V}$ to $-3 \mathrm{~V}$. (B) $I-V$ characteristics of the ITO/PI-NT amorphous film/LiF/Al on the negative scan. Sweep 1: from $0 \mathrm{~V}$ to $-3 \mathrm{~V}$; sweeps 2: from $-3 \mathrm{~V}$ to $0 \mathrm{~V}$; sweeps 3 and 4: reversible sweep between $0 \mathrm{~V}$ to $+3 \mathrm{~V}$; sweeps 5 and 6 : reversible sweep between 0 $\mathrm{V}$ to $-3 \mathrm{~V}$. The PI-NT amorphous films were synthesized in DMF at $180{ }^{\circ} \mathrm{C}$ and were confirmed by ART-FTIR spectroscopy and GIWAXS patterns. 
(a)
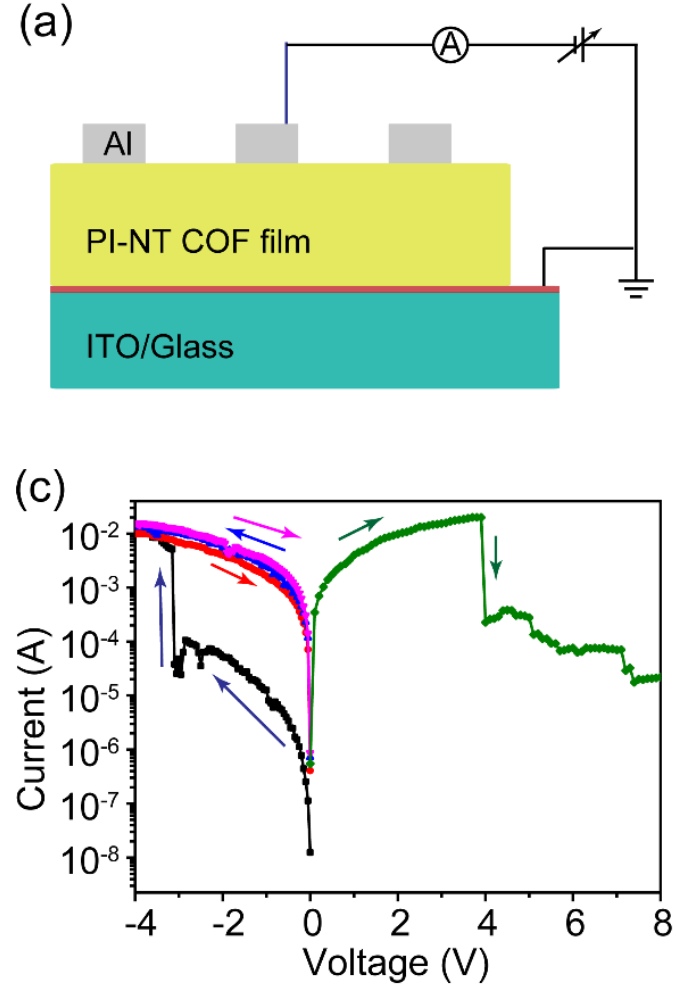

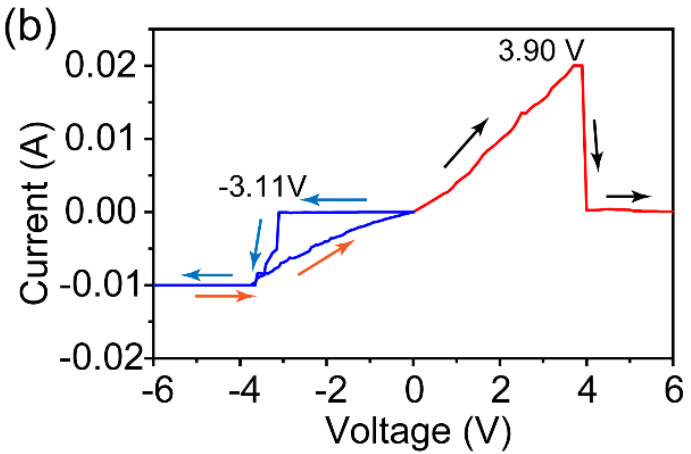

(d)

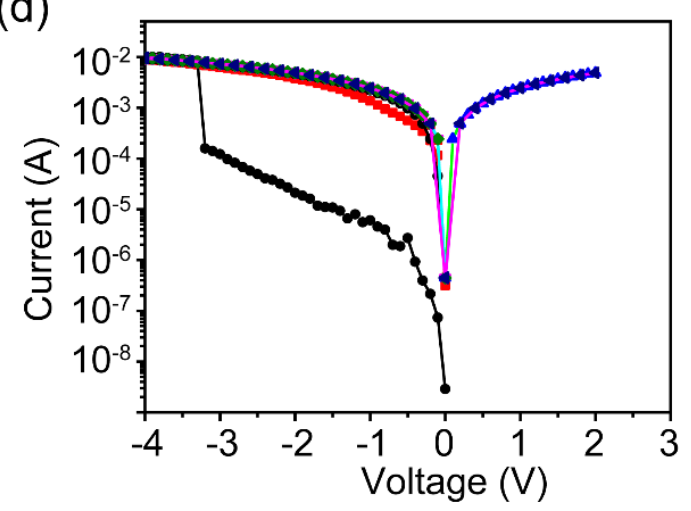

Figure S16. (a) Illustration of the resistive switching memory device for ITO/PI-NT COF film/Al configuration. (b) $I-V$ characteristics in linear current plot for ITO/PI-NT COF film/Al devices in the initial formation process and corresponding (c) $I-V$ characteristics in logarithmic current plot of the SET, READ and RESET process. The arrows represented the voltage sweeping direction. (d) $I-V$ characteristics manipulated as the forward voltage in the range of $-4 \mathrm{~V}$ and $+2 \mathrm{~V}$ for ITO/PI-NT COF film/Al devices. 
S4. Supplementary characterization for PI-NT COF and COF-based memory devices

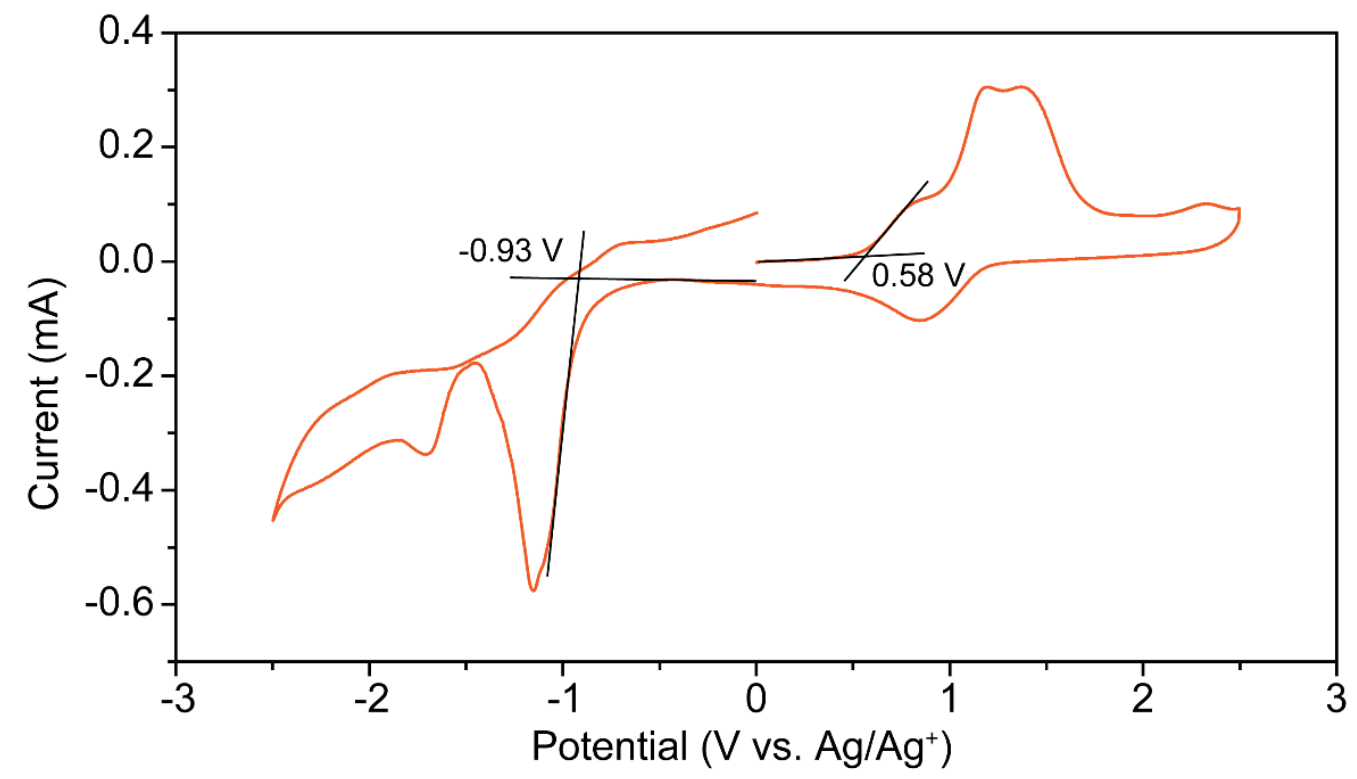

Figure S17. Cyclic voltammetry diagram of PI-NT COF films grown on ITO-coated glass substrate employing anhydrous acetonitrile $\left(10 \mathrm{~mL}\right.$, containing $\left.0.1 \mathrm{M} \mathrm{Bu}_{4} \mathrm{NPF}_{6}\right)$ as electrolyte at a scan rate of $50 \mathrm{mV} \mathrm{s}^{-1}$.

The on-set reduce potential $\left(E_{\mathrm{re}}{ }^{\text {on-set }}\right)$ was $-0.93 \mathrm{~V}$ and the on-set oxidation potential $\left(E_{\text {ox }}{ }^{\text {on-set }}\right)$ was $+0.58 \mathrm{~V}$.

The LUMO and HOMO position can be calculated as:

$$
\begin{aligned}
& E_{\mathrm{LUMO}}=-\left[\left(E_{\mathrm{re}}{ }^{\text {on-set }}-E_{\mathrm{re}}{ }^{\mathrm{Fc}}+4.8\right] \mathrm{eV}\right. \\
& E_{\mathrm{HOMO}}=-\left[\left(E_{\text {ox }}{ }^{\text {on-set }}-E_{\text {ox }}{ }^{\mathrm{Fc}}+4.8\right] \mathrm{eV}\right.
\end{aligned}
$$

Thus, $E_{\mathrm{LUMO}}=-3.62 \mathrm{eV}, E_{\mathrm{HOMO}}=-5.09 \mathrm{eV}$, and $E_{\mathrm{g}}=1.47 \mathrm{eV}$. 


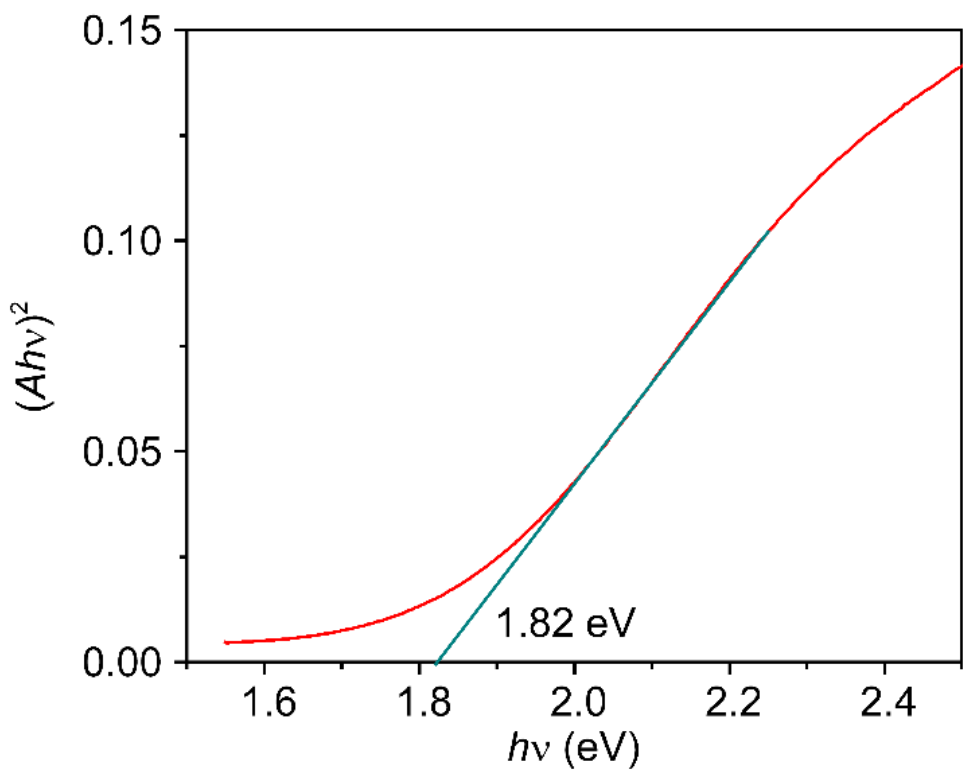

Figure S18. Tauc plot deriving from the UV-visible light absorption spectrum. The bandgap $\left(E_{\mathrm{g}}\right)$ was calculated to be $1.82 \mathrm{eV}$.
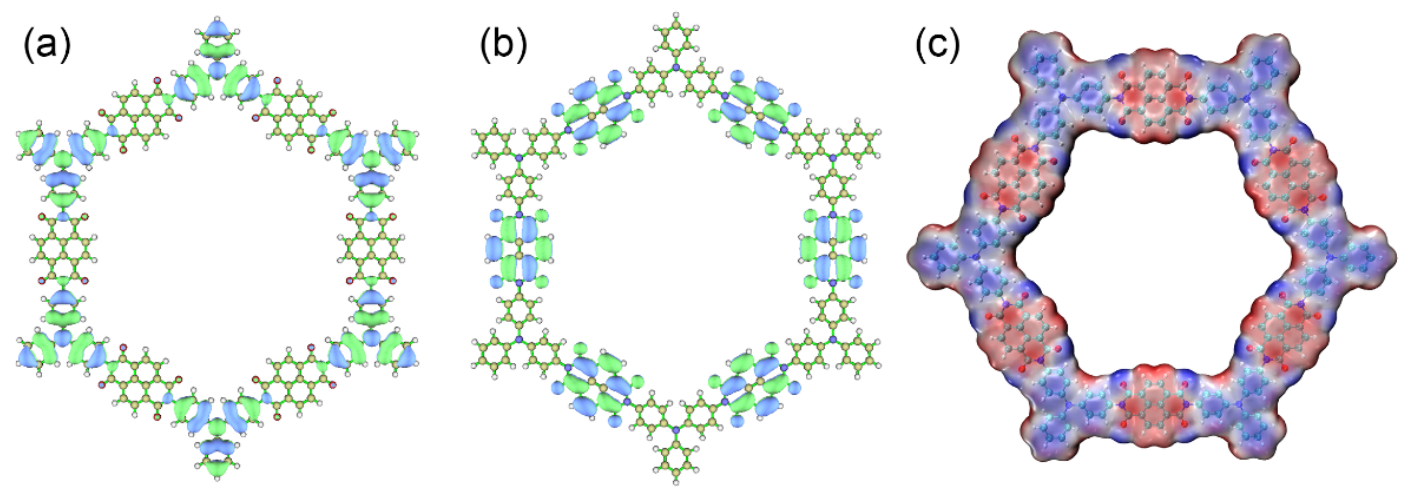

Figure S19. The frontier orbitals (a) HOMO, (b) LUMO and (c) electrostatic potential of PI-NT COF fragment.

The frontier molecular orbital distributions of PI-NT COF fragment were optimized and calculated based upon Density Functional Theory (DFT) using the Gaussian09 software package. ${ }^{\text {S17 }}$ The RB3LYP function with 6-311G(d, p) basis set was documented to be computationally accurate and fast for molecular orbital energy gap and frontier obital calculations. During the calculations, the cleaved bonds of the ring in the fragments models were terminated by hydrogen atoms and the molecular symmetries were kept as $D 3 d$ and $S 6$ respectively. 


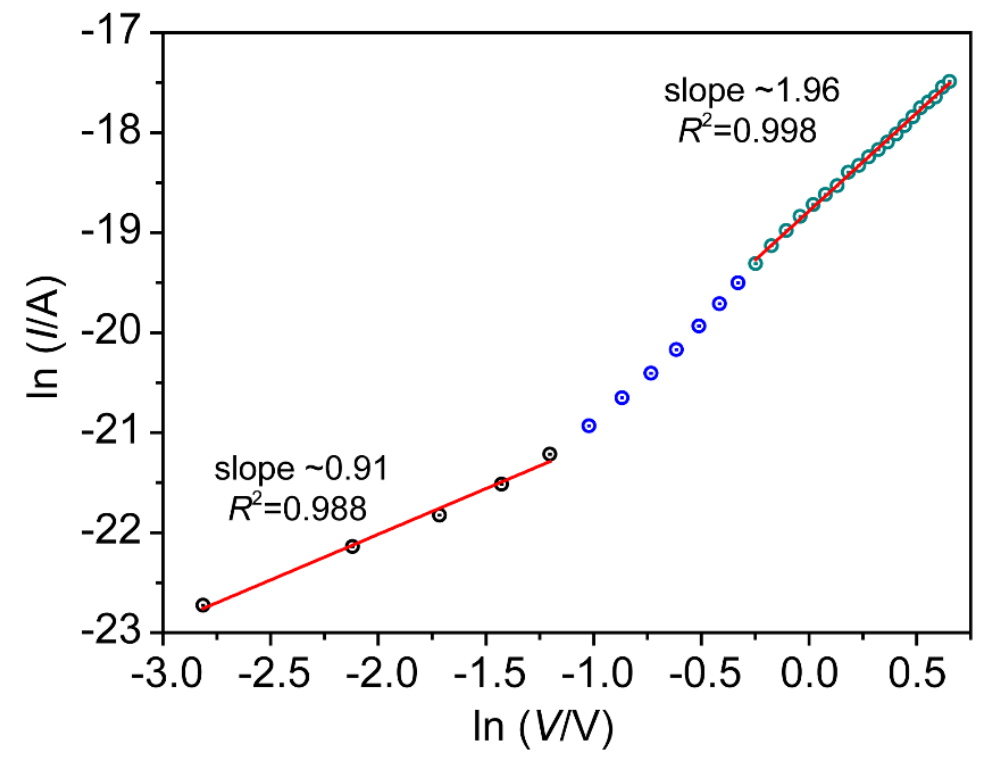

Figure S20. Space-charge limited-current (SCLC) fitting plot in logarithmic on the positive scan.

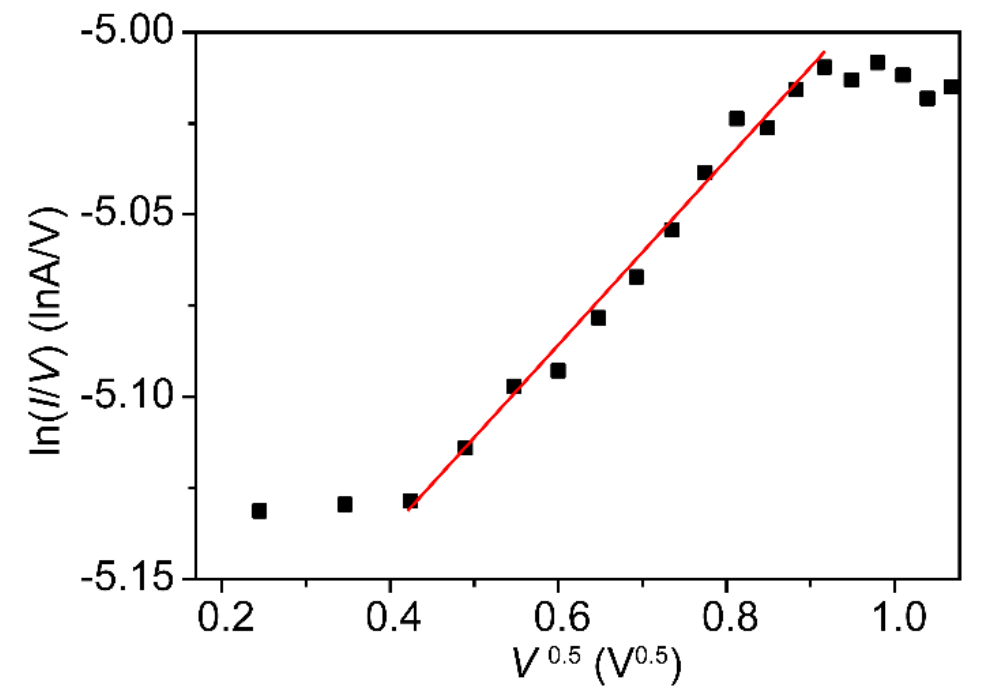

Figure S21. Poole-Frenkel (PF) emission fitting plot in the LRS on the positive scan. 


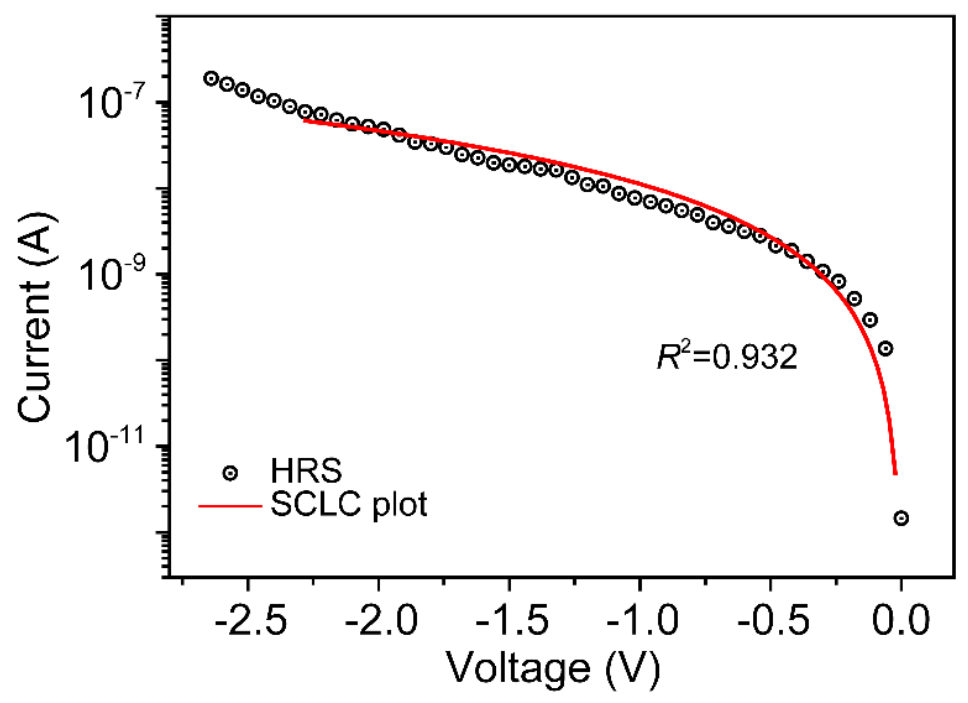

Figure S22. Space-charge limited-current (SCLC) fitting plot as scanning from $0 \mathrm{~V}$ to $-2.6 \mathrm{~V}$ in the HRS.

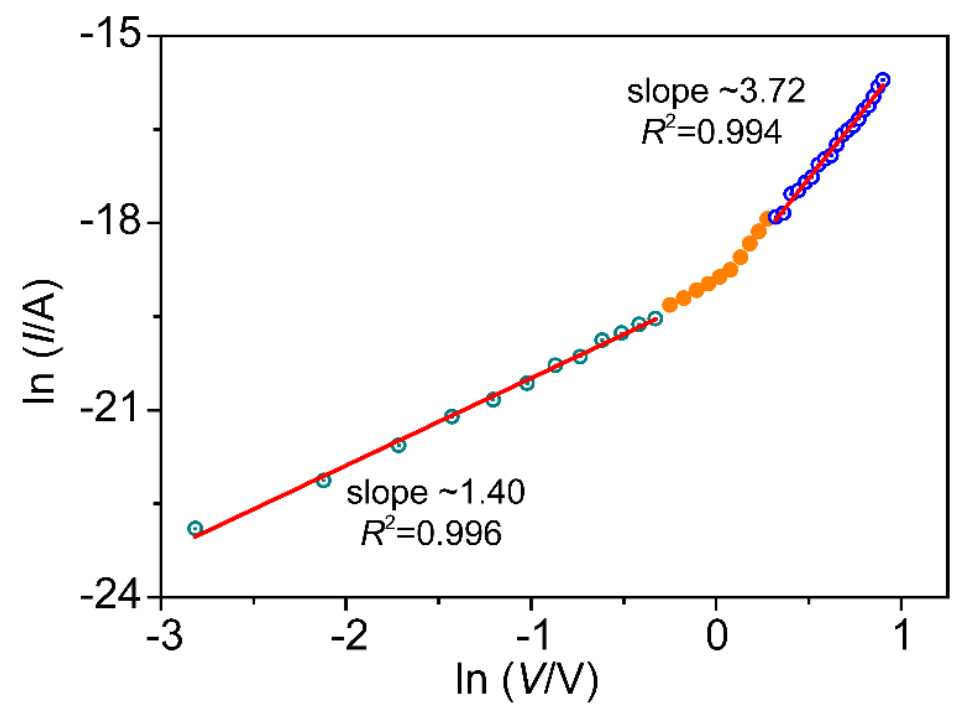

Figure S23. Space-charge limited-current (SCLC) fitting plot in logarithmic on the negative scan. 


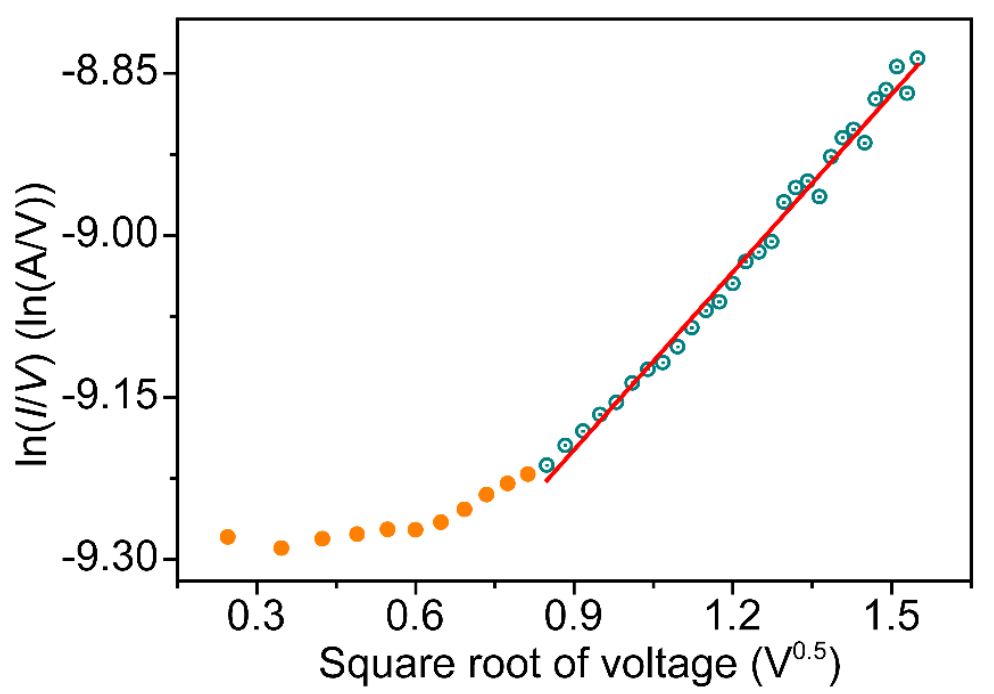

Figure S24. Poole-Frenkel (PF) emission fitting plot in the LRS on the negative scan.

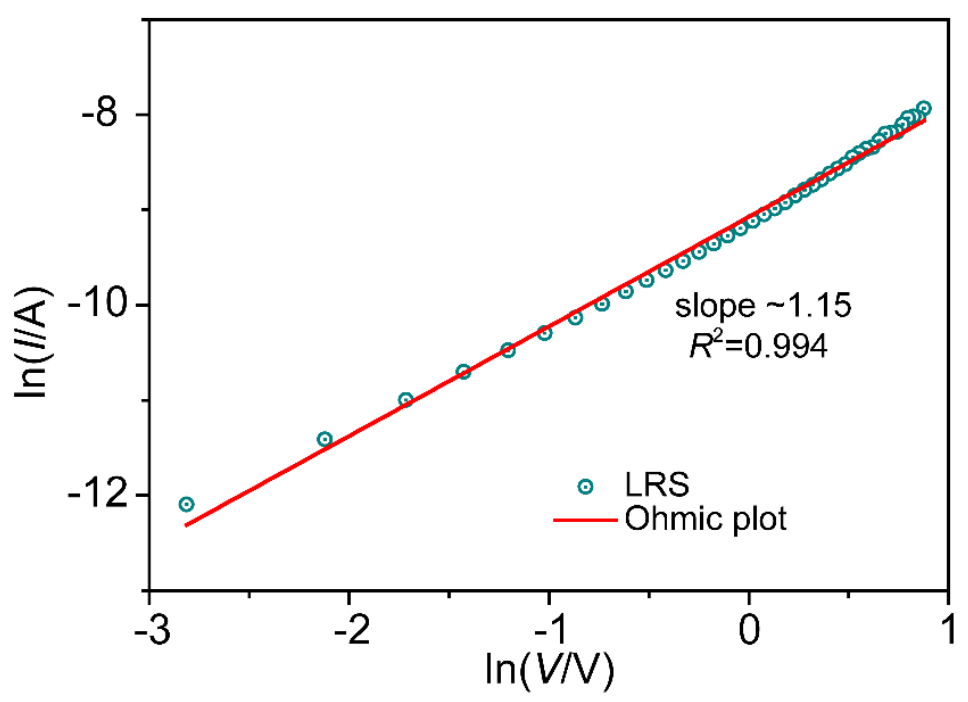

Figure S25. $\ln I v s . \ln V$ plot in the LRS on the negative scan. 
Table S3. Fractional atomic coordinates for the unit cell of PI-NT COF (Eclipsed) Space group: $P-31 m$ (162) - trigonal

Cell parameters: $a=b=32.2430 \AA, c=4.2758 \AA, c / a=0.1326 ; \alpha=\beta=90^{\circ}, \gamma=120^{\circ}$; $V=3849.63 \AA^{3}$

\begin{tabular}{|c|c|c|c|c|c|}
\hline No. & Element & Symbol & $\mathbf{a}$ & $\mathbf{b}$ & c \\
\hline 1 & $\mathrm{C}$ & $\mathrm{C} 1$ & 0.40399 & 0.74497 & -0.15591 \\
\hline 2 & $\mathrm{C}$ & $\mathrm{C} 2$ & 0.42929 & 0.79508 & -0.15285 \\
\hline 3 & $\mathrm{C}$ & $\mathrm{C} 3$ & 0.41639 & 0.89632 & -0.14636 \\
\hline 4 & $\mathrm{C}$ & $\mathrm{C} 4$ & 0.44240 & 0.94949 & -0.15027 \\
\hline 5 & $\mathrm{C}$ & $\mathrm{C} 5$ & 0.42308 & 0.97497 & -0.30234 \\
\hline 6 & $\mathrm{O}$ & O6 & 0.37747 & 0.87423 & -0.27809 \\
\hline 7 & $\mathrm{H}$ & $\mathrm{H} 7$ & 0.41970 & 0.72668 & -0.27576 \\
\hline 8 & $\mathrm{H}$ & $\mathrm{H} 8$ & 0.46386 & 0.81466 & -0.26944 \\
\hline 9 & $\mathrm{H}$ & H9 & 0.38855 & 0.95579 & -0.42131 \\
\hline 10 & $\mathrm{C}$ & $\mathrm{C} 10$ & 0.35945 & 0.71891 & 0.00000 \\
\hline 11 & $\mathrm{C}$ & $\mathrm{C} 11$ & 0.41020 & 0.82039 & 0.00000 \\
\hline 12 & $\mathrm{~N}$ & N12 & 0.43593 & 0.87186 & 0.00000 \\
\hline 13 & $\mathrm{C}$ & $\mathrm{C} 13$ & 0.48734 & 0.97468 & 0.00000 \\
\hline 14 & $\mathrm{~N}$ & N14 & 0.33333 & 0.66667 & 0.00000 \\
\hline
\end{tabular}


Table S4. Fractional atomic coordinates for the unit cell of PI-NT COF (Staggered) Space group: $P-31 c(163)$ - trigonal

Cell parameters: $a=b=32.1590 \AA, c=7.7957 \AA, c / a=0.2424 ; \alpha=\beta=90^{\circ}, \gamma=120^{\circ}$; $V=6982.18 \AA^{3}$

\begin{tabular}{|c|c|c|c|c|c|}
\hline No. & Element & Symbol & $\mathbf{a}$ & b & c \\
\hline 1 & $\mathrm{C}$ & $\mathrm{C} 1$ & 0.07112 & 1.07839 & 0.16848 \\
\hline 2 & $\mathrm{C}$ & $\mathrm{C} 2$ & 0.09644 & 1.12858 & 0.16992 \\
\hline 3 & $\mathrm{C}$ & $\mathrm{C} 3$ & 0.08401 & 1.22976 & 0.16494 \\
\hline 4 & $\mathrm{C}$ & $\mathrm{C} 4$ & 0.10984 & 1.28307 & 0.16460 \\
\hline 5 & $\mathrm{C}$ & $\mathrm{C} 5$ & 0.09094 & 1.30862 & 0.08033 \\
\hline 6 & $\mathrm{O}$ & O6 & 0.04606 & 1.20751 & 0.08776 \\
\hline 7 & $\mathrm{H}$ & $\mathrm{H} 7$ & 0.08678 & 1.06006 & 0.10296 \\
\hline 8 & $\mathrm{H}$ & H8 & 0.13123 & 1.14818 & 0.10802 \\
\hline 9 & $\mathrm{H}$ & H9 & 0.05679 & 1.28939 & 0.01381 \\
\hline 10 & $\mathrm{C}$ & $\mathrm{C} 10$ & 0.26578 & 0.58923 & 0.34995 \\
\hline 11 & $\mathrm{C}$ & $\mathrm{C} 11$ & 0.24049 & 0.53897 & 0.34892 \\
\hline 12 & $\mathrm{C}$ & $\mathrm{C} 12$ & 0.25038 & 0.43771 & 0.33067 \\
\hline 13 & $\mathrm{C}$ & $\mathrm{C} 13$ & 0.22415 & 0.38438 & 0.33370 \\
\hline 14 & $\mathrm{C}$ & $\mathrm{C} 14$ & 0.24297 & 0.35883 & 0.41844 \\
\hline 15 & $\mathrm{O}$ & $\mathrm{O} 15$ & 0.28935 & 0.45996 & 0.40121 \\
\hline 16 & $\mathrm{H}$ & H16 & 0.25312 & 0.60834 & 0.42884 \\
\hline 17 & $\mathrm{H}$ & H17 & 0.20824 & 0.51935 & 0.42501 \\
\hline 18 & $\mathrm{H}$ & $\mathrm{H} 18$ & 0.27731 & 0.37804 & 0.48378 \\
\hline 19 & $\mathrm{C}$ & C19 & 0.02615 & 1.05231 & 0.25000 \\
\hline 20 & $\mathrm{C}$ & $\mathrm{C} 20$ & 0.07692 & 1.15383 & 0.25000 \\
\hline
\end{tabular}




\begin{tabular}{llllll}
\hline 21 & $\mathrm{~N}$ & $\mathrm{~N} 21$ & 0.10266 & 1.20532 & 0.25000 \\
22 & $\mathrm{C}$ & $\mathrm{C} 22$ & 0.15417 & 1.30833 & 0.25000 \\
23 & $\mathrm{C}$ & $\mathrm{C} 23$ & 0.30741 & 0.61481 & 0.25000 \\
24 & $\mathrm{C}$ & $\mathrm{C} 24$ & 0.25684 & 0.51368 & 0.25000 \\
25 & $\mathrm{~N}$ & $\mathrm{~N} 25$ & 0.23108 & 0.46215 & 0.25000 \\
26 & $\mathrm{C}$ & $\mathrm{C} 26$ & 0.17956 & 0.35912 & 0.25000 \\
27 & $\mathrm{~N}$ & $\mathrm{~N} 27$ & 0.00000 & 1.00000 & 0.25000 \\
28 & $\mathrm{~N}$ & $\mathrm{~N} 28$ & 0.33333 & 0.66667 & 0.25000 \\
\hline
\end{tabular}




\section{References}

S1.Zhang, Q.; He, J.; Zhuang, H.; Li, H.; Li, N.; Xu, Q.; Chen, D.; Lu, J. Rational Design of Small Molecules to Implement Organic Quaternary Memory Devices. $A d v$. Funct. Mater. 2016, 26, 146-154.

S2.Khan, Q. U.; Lu, J.; Guo, J.; Tian, G.; Qi, S.; Wu, D. Synthesis of linear, V-shaped and Star-shaped Asymmetrical Perylene Diimides Bearing Triphenylamine Moiety for Resistive Memory Application. Dyes Pigm. 2018, 149, 193-200.

S3.Wu, H.-C.; Zhang, J.; Bo, Z.; Chen, W.-C. Well-defined Star-shaped DonorAcceptor Conjugated Molecules for Organic Resistive Memory Devices. Chem. Commun. 2015, 51, 14179-14182.

S4.Wang, C.; Wang, J.; Li, P. Z.; Gao, J.; Tan, S. Y.; Xiong, W. W.; Hu, B.; Lee, P. S.; Zhao, Y.; Zhang, Q. Synthesis, Characterization, and Non-volatile Memory Device Application of an N-substituted Heteroacene. Chem. Asian J. 2014, 9, 779-783.

S5.Wang, C.; Yamashita, M.; Hu, B.; Zhou, Y.; Wang, J.; Wu, J.; Huo, F.; Lee, P. S.; Aratani, N.; Yamada, H.; Zhang, Q. Synthesis, Characterization, and Memory Performance of Two Phenazine/Triphenylamine-based Organic Small Molecules through Donor-Acceptor Design. Asian J. Org. Chem. 2015, 4, 646-651.

S6.Pan, L.; Hu, B.; Zhu, X.; Chen, X.; Shang, J.; Tan, H.; Xue, W.; Zhu, Y.; Liu, G.; Li, R.-W. Role of Oxadiazole Moiety in Different D-A Polyazothines and Related Resistive Switching Properties. J. Mater. Chem. C 2013, 1, 4556-4564

S7.Xiang, J.; Wang, T.-K.; Zhao, Q.; Huang, W.; Ho, C.-L.; Wong, W.-Y. FerroceneContaining Poly(fluorenylethynylene)s for Nonvolatile Resistive Memory Devices. J. Mater. Chem. C 2016, 4, 921-928.

S8.Yu, A.-D.; Kurosawa, T.; Lai, Y.-C.; Higashihara, T.; Ueda, M.; Liu, C.-L.; Chen, W.-C. Flexible Polymer Memory Devices Derived from Triphenylamine-Pyrene Containing Donor-Acceptor Polyimides. J. Mater. Chem. 2012, 22, 20754-20763.

S9.Wang, K.-L.; Liu, G.; Chen, P.-H.; Pan, L.; Tsai, H.-L. Structural Effect on Controllable Resistive Memory Switching in Donor-Acceptor Polymer Systems. Org. Electron. 2014, 15, 322-336.

S10. Yu, A. D.; Kurosawa, T.; Chou, Y. H.; Aoyagi, K.; Shoji, Y.; Higashihara, T.; Ueda, M.; Liu, C. L.; Chen, W. C. Tunable Electrical Memory Characteristics Using Polyimide: Polycyclic Aromatic Compound Blends on Flexible Substrates. ACS Appl. Mater. Interfaces 2013, 5, 4921-4929.

S11. Tian, Y.; Song, Y.; Yao, H.; Yu, H.; Tan, H.; Song, N.; Shi, K.; Zhang, B.; Zhu, S.; Guan, S. Improving Resistive Switching Characteristics of Polyimide-based Volatile Memory Devices by Introducing Triphenylamine Branched Structures. Dyes Pigm. 2019, 163, 190-196.

S12. Hsu, L.-C.; Shih, C.-C.; Hsieh, H.-C.; Chiang, Y.-C.; Wu, P.-H.; Chueh, C.-C.; Chen, W.-C. Intrinsically Stretchable, Solution-Processable Functional Poly(siloxane-imide)s for Stretchable Resistive Memory Applications. Polym. Chem. 2018, 9, 5145-5154.

S13. Qu, L.; Tang, L.; Bei, R.; Zhao, J.; Chi, Z.; Liu, S.; Chen, X.; Aldred, M. P.; Zhang, Y.; Xu, J. Flexible Multifunctional Aromatic Polyimide Film: Highly Efficient Photoluminescence, Resistive Switching Characteristic, and 
Electroluminescence. ACS Appl. Mater. Interfaces 2018, 10, 11430-11435.

S14. Tsai, M.-C.; Wang, C.-L.; Lin, C.-Y.; Tsai, C.-L.; Yen, H.-J.; You, H.-C.; Liou, G.-S. A novel porphyrin-containing polyimide for memory devices. Polym. Chem. 2016, 7, 2780-2784.

S15. Fu, L.; Zhang, Y.; Ren, Z.; Li, H.; Sun, X.; Yan, S. Flexible and Fatigue-

Resistant Ternary Electrical Memory Based on Alternative Copolysiloxane with Carbazole Donors and Imidazole-Modified Naphthalimide Acceptors. Adv. Mater. Technol. 2019, 4, 1900084.

S16. Liu, J.; Yang, F. X.; Cao, L. L.; Li, B. L.; Yuan, K.; Lei, S. B.; Hu, W. P. A

Robust Nonvolatile Resistive Memory Device Based on a Freestanding Ultrathin 2D Imine Polymer Film. Adv. Mater. 2019, 31, e1902264.

S17. M. J. Frisch, G. W. Trucks, H. B. Schlegel, G. E. Scuseria, M. A. Robb, J. R. Cheeseman, G. Scalmani, V. Barone, B. Mennucci, G. A. Petersson, H. Nakatsuji, M. Caricato, X. Li, H. P. Hratchian, A. F. Izmaylov, J. Bloino, G. Zheng, J. L. Sonnenberg, M. Hada, M. Ehara, K. Toyota, R. Fukuda, J. Hasegawa, M. Ishida, T. Nakajima, Y. Honda, O. Kitao, H. Nakai, T. Vreven, J. A. Montgomery, Jr., J. E. Peralta, F. Ogliaro, M. Bearpark, J. J. Heyd, E. Brothers, K. N. Kudin, V. N. Staroverov, T. Keith, R. Kobayashi, J. Normand, K. Raghavachari, A. Rendell, J. C. Burant, S. S. Iyengar, J. Tomasi, M. Cossi, N. Rega, J. M. Millam, M. Klene, J. E. Knox, J. B. Cross, V. Bakken, C. Adamo, J. Jaramillo, R. Gomperts, R. E. Stratmann, O. Yazyev, A. J. Austin, R. Cammi, C. Pomelli, J. W. Ochterski, R. L. Martin, K. Morokuma, V. G. Zakrzewski, G. A. Voth, P. Salvador, J. J. Dannenberg, S. Dapprich, A. D. Daniels, O. Farkas, J. B. Foresman, J. V. Ortiz, J. Cioslowski, and D. J. Fox, Gaussian 09, Revision D.01, Gaussian, Inc., Wallingford CT, 2013. 